\title{
Palmoplantar pustulosis secondary to anti-tumor necrosis factor therapy in a patient with ankylos- ing spondylitis
}

Jung Min Bae ${ }^{1}$, Sung Hye Eun ${ }^{1}$, and Ki-Jo Kim²

${ }^{1}$ Department of Dermatology,

${ }^{2}$ Division of Rheumatology, Department of Internal Medicine, College of Medicine, St. Vincent's Hospital, The Catholic University of Korea, Suwon, Korea
Received: June 5, 2018

Revised : June 15, 2018

Accepted: July 6, 2018

\section{Correspondence to}

Ki-Jo Kim, M.D.

Tel: +82-31-249-8156

Fax: +82-31-253-8898

E-mail:md21c@catholic.ac.kr
A 34-year-old man presented with crops of pustules with erythematous scaly patches on his soles and palms (Fig. 1A-1D). Both great fingernails showed trachyonychia and onycholysis with transverse ridges (Fig. 1E). He had a 6-year history of ankylosing spondylitis manifested as positive human leukocyte antigen B27 (HLA-B27) and bilateral sacroiliitis. In the radiographs
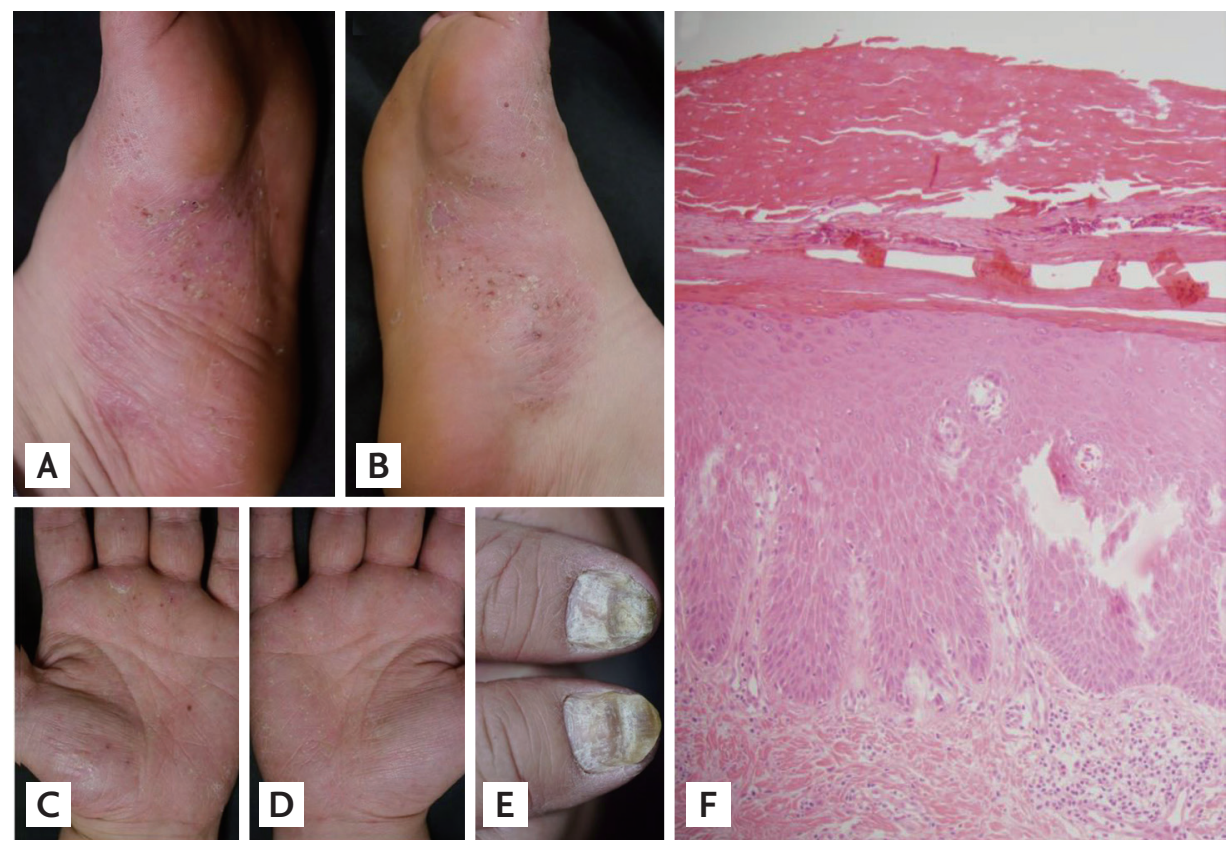

Figure 1. Clinical features of palmoplantar pustulosis after anti-TNF therapy and a radiograph of the lumbar spine of the patient. Crops of small, deep-seated pustules with diffuse, erythematous scaly patches on the patient's soles (A, B) and palms (C, D). Trachyonychia and onycholysis with transverse ridges on both great fingernails (E). Skin biopsy from the palm revealed hyperkeratosis, parakeratosis, and a pustule in the corneal layer and spongiosis and perivascular lymphocytic infiltration in the upper (F: H\&E stain, $\times 200)$. 


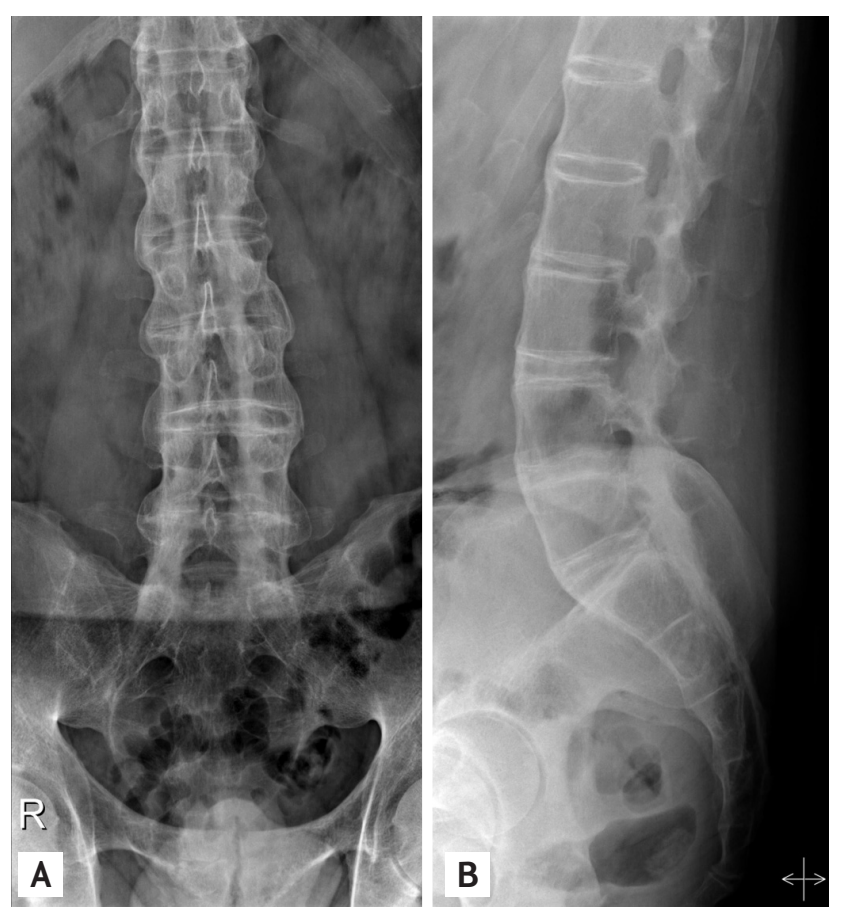

Figure 2. Plain lumbar radiograph of the patient shows bamboo spine and complete fusion of sacroiliac joints. (A) Anteroposterior view, (B) lateral view.

tule in the corneal layer and spongiosis and perivascular lymphocytic infiltration in the upper dermis, consistent with psoriasiform dermatitis (Fig. $1 F$ ). Palmoplantar pustulosis secondary to adalimumab was suspected.
After discontinuing the adalimumab, the skin lesions improved with topical corticosteroid and calcipotriol ointment. Two months later, etanercept was administered to control a flare of ankylosing spondylitis, and no recurrence has been noted for 2 years. Anti-tumor necrosis factor (TNF) agents are widely used in the management of various inflammatory disorders, including inflammatory bowel disease, rheumatoid arthritis, and ankylosing spondylitis. Growing numbers of clinical observations have described the paradoxical incidence or exacerbation of psoriasis in patients treated with anti-TNF agents, although the agents are also effective for psoriasis itself. Unopposed activation of the interferon- $\alpha$ pathway following neutralization of TNF has been suggested as the possible mechanism. A class effect is proposed for this paradoxical event, but other anti-TNF agents could be cautiously tried because the magnitude of the effect across anti-TNF agents could be different.

This study was carried out in accordance with the Declaration of Helsinki and approved by the St. Vincent's Hospital Institutional Review Board (IRB) (No. VC18ZESIo122). Written informed consent was waived because this report does not contain any personally identifiable information.

\section{Conflict of interest}

No potential conflict of interest relevant to this article was reported. 